\title{
A NSW PUBLIC HEALTH LABORATORY NETWORK
}

John Rooney

formerly Specialist Medical Adviser

AIDS / Infectious Diseases Branch, NSW Health Department

$T_{\text {Hed }}^{\text {h }}$

his article outlines a proposal to establish a Public

Health Laboratory Network (PHLN) in NSW, designed to meet requirements for high quality public health laboratory services and laboratory expertise by building on existing services and facilities. The proposed PHLN, with the State's network of Public Health Units and the NSW Health Department's AIDS/Infectious Diseases and Environmental Health, Food and Nutrition Branches, will provide an integrated public health service for the surveillance and control of infectious diseases, and the capacity to monitor the safety of food and water and the risk of harm from exposure to toxic substances in the environment.

The PHLN will comprise a network of public health laboratories formally designated to provide specified services. Individual laboratories will represent foci of Statewide expertise in specific public health areas, while in combination the network will give NSW a comprehensive public health laboratory service.

\section{THE CURRENT SITUATION}

Public health laboratory services in NSW are provided through a variety of arrangements.

- Many public and private clinical laboratories in NSW are involved in public health surveillance through the requirement to notify certain infectious diseases under the Public Health Act 1991. Most of these notifications arise from clinical diagnostic testing for individual patient management. Some laboratories also contribute to public health through the analysis of food, water and environmental samples as well as human samples, both for research and in the investigation of disease outbreaks.

An informal ad hoc network of specialist

laboratories, managed by a number of different Area Health Services, provides expertise in specific public health fields.

Specialist university, research and private

laboratories also provide expertise in specific public health fields.

Although these arrangements have worked satisfactorily to date, the requirements of a modern, responsive and efficient public health service dictate a more formal and explicit structure.

Recent developments in the NSW Health system favour such a structure. Responsibility for the Division of Analytical Laboratories has been devolved from the NSW Health Department to the Western Sydney Area Health Service, and the microbiology and virology laboratories of the Institute of Clinical Pathology and Medical Research at Westmead Hospital have been integrated. Thus several significant Statewide public health functions have come together within the Western Sydney Area. The creation of the South Eastern Area Laboratory Service and the rationalisation of pathology services within other Areas are also likely to lead to new opportunities for laboratories to acquire or expand Statewide roles in certain fields of expertise.
TABLE 1

ROLES OF DESIGNATED PUBLIC HEALTH LABORATORIES

- Capacity to respond to Statewide public health requirements, including outbreak investigations, in conjunction with the NSW Health Department.

- Policy development (e.g. as a result of changing patterns of antibiotic resistance).

- Education (e.g. the development and updating of testing algorithms for general practitioners and public health practitioners).

- Providing a Statewide and/or national reference laboratory function.

- Coordinating the NSW contribution to State, national and international laboratory networks to facilitate the collation and analysis of epidemiological data.

- Analysis and reporting of Statewide data in conjunction with the NSW Health Department.

- Providing advice on changes in technology and opportunities for improved efficiency in the laboratory contribution to public health investigations.

- Training and advice (e.g. in the identification of malarial parasites, vectors for arboviral infection).

- Quality assurance (coordinating Statewide response to State, national and international programs).

- In conjunction with other laboratories and the Department the development of benchmarks and best practice for public health laboratory surveillance and investigation.

- Developing case definitions and standardised reporting formats for notifiable conditions.

- Ensuring the availability of techniques for low volume requests in a way which avoids duplication by offering the service on site or making sure any request can be appropriately referred.

- Receipt of isolates and samples for confirmatory testing, typing, or archiving (either as a voluntary role or as a required public health responsibility).

- Providing a consultancy service to Public Health Units and the NSW Health Department.

- Initiating public health research in conjunction with the Department, Public Health Units and other agencies.

- Conducting and/or coordinating seroprevalence and other surveys in response to Statewide public health priorities determined by the Department.

- Providing specialist input into the Public Health Laboratory Advisory Committee.

THE CONCEPT OF DESIGNATED LABORATORIES The intent of the PHLN is to identify designated laboratories for the Department, public health practitioners and external agencies. Designated laboratories may provide generic or specialist services. Through the PHLN there will be a known point of contact for the laboratory services needed to support any Statewide public health contingency, including routine and planned public health action, the investigation of outbreaks, and the response to emergencies and disasters which pose a threat to public health. The PHLN does not seek to provide a neat bureaucratic framework, but rather a mechanism for facilitating communication between laboratories, clinicians, public health practitioners and managers. 


\section{Public Health Laboratory Network}

\section{Continued from page 25}

The concept of designation implies neither pre-eminence nor an exclusive role in testing. In some instances (e.g. testing for malaria), laboratories will have exclusive roles. In other instances a laboratory is selected to have a coordinating role and to provide advocacy and support for other laboratories which will continue to provide confirmatory and/or reference functions and contributions to research.

Table 1 lists generic functions for designated laboratories. Some designated laboratories will take on all these functions, while others will fulfil only some generic functions. The functions to be undertaken by each designated laboratory will be negotiated between the NSW Health Department and the Area Health Service managing the laboratory, and will clearly be influenced by available expertise and resources.

Three approaches may be taken to the designation of laboratories for specialist public health functions.

First, laboratories may be designated for a single disease or organism. This approach is likely to be used for uncommon but important conditions. Examples are:

- malaria, where the laboratory has a specific public health role;

- Legionella infection, where responsibility must be clear-cut in the event of an outbreak; and - tuberculosis, which has specific public health implications, with the laboratory role in monitoring antibiotic resistance being of crucial importance.

- Second, laboratories may be designated for a group of diseases or organisms. This may be more helpful for public health practice than designation for a single disease or organism. Vaccinepreventable diseases (diphtheria, Haemophilus influenzae, measles, mumps, pertussis,

Pneumococcus, rubella and varicella) have been grouped because designated laboratories may have particular roles, e.g. in coordinating prevalence surveys to monitor the effectiveness of immunisation programs.

- Designating a laboratory for a specific public health function is the third approach which has been used. Water quality and food safety are examples of such functions.

In some instances a designated laboratory's roles will include collating results of tests routinely carried out in most laboratories (e.g. diagnosis of Neisseria infection). For others the designated laboratory will be one of a small number approved for confirmatory testing (e.g. for HIV infection). In a few instances the designated laboratory may have a State or national reference function, and the laboratory may then be widely accepted as the sole focus of public health expertise (e.g. medical entomology and water quality assessment).

\section{SELECTION OF DESIGNATED LABORATORIES}

For most laboratories the selection has been based on existing roles and generally accepted views about the current focus of expertise. However, because not all

\section{TABLE 2}

DESIGNATED PUBLIC HEALTH LABORATORY (TO DECEMBER 1997) Condition/s

Designated laboratory (abbreviations are given below)

Arboviral diseases'

Chemicals in the environment ${ }^{2}$

Enteric diseases ${ }^{3}$

ICPMR

Food safety ${ }^{4}$

Hepatitis

Influenza

HIV/AIDS

Legionella

Medical entomology (other

than arboviral diseases)

Malaria (and parasitic diseases

other than giardiasis

and cryptosporidium)

Neisseria ${ }^{5}$

Quarantinable and other rare, exotic, or imported conditions ${ }^{6}$

Vaccine preventable diseases

STDs $^{8}$

DAL/ICPMR

ICPMR/DAL

DALICPMR

Not determined

SEALS

St Vincent's/SEALS

ICPMR/DAL

ICPMR

ICPMR

SEALS/SWAPS

ICPMR

SEALS/ICPMR

ICPMR

Tuberculosis and other

mycobacteria

Water quality

Zoonotic diseases $^{9}$

ICPMR

DAL/ICPMR

ICPMR

$\mathrm{DAL}=$ Division of Analytical Laboratories; ICPMR = Institute of Clinical Pathology \& Medical Research, Westmead Hospital; St Vincent's = St Vincent's Hospital, Darlinghurst; SEALS = South East Area Laboratory Service; SWAPS = South West Area Pathology Service.

1. Including Dengue, Ross River Fever, Barmah Forest Disease, Murray Valley Encephalitis, other Alpha, Bunya and Flaviviruses, Exotic and VHF viruses.

2. Including pesticides, trace metals.

3. Including viral (rotavirus), bacterial (Campylobacter, salmonella, shigella, yersinia, cholera, e coli...) Parasital (cryptosporidium). 4. Including listeriosis, botulism.

5. Including gonorrhoea, meningococcal disease.

6. Including cholera, plague, rabies, typhus, viral haemorrhagic fevers,

vellow fever, equine morbilliform virus.

7. Including diphtheria, haemophilus influenzae, measles, mumps,

pneumococcus, poliomyelitis rubella, varicella.

8. Including chancroid, chlamydia trachomatis, donovanosis, herpes, syphilis.

9. Including brucellosis, hydatid disease, leptospirosis, Q fever.

interested parties could be consulted in the development of the PHLN, it has been suggested that designation of laboratories should apply for 12 months only in the first instance. This permits the rationale for selection to be challenged before designation is offered for five years.

Table 2 lists designated public health laboratories and recommended sites for the initial 12-month period (to the end of December 1997).

\section{COORDINATION OF THE PHLN}

It is envisaged that the PHLN will become part of the wider NSW Public Health Network; specifically, that it will have close links with the NSW Health Department and Area Health Services through the Public Health Units.

The Department (through the Chief Health Officer) is responsible for public health policy and for ensuring 
Although the incubation period for rabies is usually 1-3 months, longer incubation periods have been reported ${ }^{3}$. Prevention of disease after exposure is effective only if PEP is administered before the onset of clinical disease. Although treatment should be initiated as soon as possible, the stage of the incubation period during which infection becomes intractable is unknown. Therefore, PEP is recommended for administration any time before the onset of symptoms, regardless of the time elapsed since exposure. RIG still may be administered for up to one week after the rabies vaccine series has been initiated. However, administration of RIG more than one week after initiation of the vaccine series is not recommended because antibodies to the virus already will have been induced by the vaccine.

\section{The Australian connection}

In this case, had the patient elected to receive PEP in Sydney, a delay of about five days would have occurred. There have been no reported failures of PEP in association with the correct implementation of the treatment regimen in the United States ${ }^{1}$, where the median interval between exposure and administration of PEP is about five days. This report highlights the importance of taking a careful exposure history when evaluating people who may have come into contact with potentially rabid animals overseas. Where such an exposure is identified, rapid administration of RIG and rabies vaccine is essential.

To arrange PEP, or where there is any doubt about its need, clinicians should seek advice from their local Public Health Unit. With the discovery of a new rabies-like lyssavirus in flying foxes and insectivorous bats in Australia and the identification of a human fatality associated with this virus in $1996^{5}$, use of PEP in NSW has increased dramatically in recent months.

\section{Travellers at risk}

The risk for rabies for international travellers is greatest in areas where canine rabies is still highly endemic, including many parts of Africa, Asia and Central and South America. Two countries where the patient in this report had extended stays - Nepal and Thailand - are considered to be areas where dog rabies is highly endemic. Pre-exposure vaccination with $\mathrm{HDCV}$ or rabies vaccine adsorbed should be considered for people living in or visiting (for greater than 30 days) areas where rabies is endemic and appropriate PEP may not be readily obtained.

Pre-exposure vaccination does not eliminate the need for additional therapy after an exposure but does simplify the post-exposure regimen by eliminating the need for RIG and decreasing the number of required vaccine doses ${ }^{6}$. Because rabies virus may be present in the saliva of infected animals 3-4 days before onset of clinical symptoms ${ }^{7}$, people who are bitten or scratched by any animal should thoroughly wash all wounds with soap and water and immediately seek medical consultation to evaluate the need for $\mathrm{PEP}^{1,2}$. Where there is a delay between a high-risk exposure and presentation for treatment, PEP should be administered regardless of the delay.

(Adapted from: CDC. Human Rabies - New Hampshire. MMWR 1997; 46:?-?.)

1. CDC. Rabies prevention - United States, 1991: recommendations of the Immunization Practices Advisory Committee (ACIP). MMWR 1991; 40(no. RR-3).

2. CDC. Human rabies - Florida, 1996. MMWR 1996; 45:719-20,727.

3. Smith JS, Fishbein DB, Rupprecht CE, Clark K. Unexplained rabies in three immigrants in the United States, a virologic investigation. N Engl J Med 1991; 324:205-11.

4. Helmick CG. The epidemiology of human rabies postexposure prophylaxis, 1980-1981. JAMA 1983; 250:1990-6.

5. Fraser GC, Hooper PT, Lunt RA et al. Encephalitis caused by a lyssavirus in fruit bats in Australia. Emerging Infectious Diseases 1996; 2:327-31.

6. CDC. Health information for international travel, 1994. Atlanta: US Department of Health and Human Services, Public Health Service, 1994: 125-8.

7. Vaughn JB Jr, Gerhardt P, Newell KW. Excretion of street rabies virus in the saliva of dogs. JAMA 1965; 193:368-8.

8. Faoagali JL, De Buse P, Strutton GM, Samaratunga H. A Case of Rabies, Med J Aust 1988; 149:702-707.

9. Grattan-Smith PJ, O'Regan WJ, Ellis PSJ, O'Flaherty SJ, MeIntyre PB, Barnes CJ. A second Australian case, with a long incubation period. Med J Aust 1992; 156:651-654.

\section{Public Health Laboratory Network}

\section{Continued from page 26}

compliance with the Public Health Act 1991 and other legislation designed to prevent illness and protect the health of the population. It is appropriate for the agenda and priorities for the network of designated public health laboratories to be determined by the Chief Health Officer.

The agenda and priorities could then be incorporated in service agreements between the Department and Area Health Services responsible for managing designated laboratories.
To assist the Chief Health Officer with the development and maintenance of the PHLN it is proposed that a Public Health Laboratory Advisory Committee be formed, with membership including balanced input from the Department, the Area Health Services, laboratories (both public and private) and Public Health Units.

\section{THE FUTURE}

It will be important for the PHLN to be reviewed constantly, taking into account rapidly changing technologies, new threats to public health, and changing priorities. This will require a willingness to terminate services no longer required, as well as a preparedness to enhance existing services and develop new services. 